Ein neues Instrument in der Klimapolitik

\section{Der Klimaschutz-Index}

\author{
Einen neuer Index vergleicht die Klimaschutz-Leistungen \\ von 53 Industrie- und Schwellenländern, die für 90 Prozent \\ des weltweiten Kohlendioxid-Ausstoßes verantwortlich sind. \\ Deutschland belegt in der Rangliste Platz 5, die USA sind \\ Vorletzter, nur Saudi-Arabien schneidet noch schlechter ab. \\ Von Jan Burck und Christoph Bals
}

D er von Germanwatch entwickelte Klimaschutz-Index soll helfen, den politischen und zivilgesellschaftlichen Druck auf diejenigen Länder zu erhöhen, die den Klimaschutz bisher vernachlässigt haben (1). Er ermöglicht einen fundierten Ländervergleich, weil er den Emissionstrend, den Emissionslevel und die Klimapolitik eines Landes berücksichtigt.

Der Emissionstrend basiert auf der Entwicklung der Emissionen in den Sektoren Energie, Verkehr, Gebäude und Industrie von 1998 bis 2003. Dabei wird berücksichtigt, dass Schwellenländer wie China und Indien industriellen Nachholbedarf haben. Der Emissionslevel ergibt sich aus dem Kohlendioxid-Ausstoß eines Landes in Relation zu Bevölkerungszahl, Bruttoinlandsprodukt und Energieverbrauch. Als dritte Komponente fließt die Bewertung der nationalen und internationalen Klimapolitik eines Landes mit ein. Durch den Index wird jährlich offen gelegt, welche Staaten ihrer Verantwortung mehr nachkommen und welche weniger.

\section{Mangel an Klimaschutz}

Der Klimaschutzindex 2006 zeigt deutlich, dass viele der Länder mit dem größten Treibhausgasausstoß ihrer Verantwortung bisher nur unzureichend nachkommen. Gleich sechs der zehn größten Kohlendioxid-Emittenten, die allein 64 Prozent der weltweiten Emissionen verursachen, sind im Index im unteren Drittel zu finden.

Der Klimaschutz-Index bewertet nicht nur die einzelnen Länder insgesamt. Er zeigt auch, wie diese Länder nach den einzelnen Kriterien abschneiden und kann somit auch als Analyse-Instrument verwendet werden.

So liegt Deutschland keineswegs überall im Spitzenbereich. Deutschland ist zwar Spitzenreiter in der internationalen Klimapolitik und konnte als einziges Industrieland seine Verkehrsemissionen leicht senken (Platz 4). Doch im Gebäudesektor liegt es nur auf Platz 31. Und während der überdurchschnittliche Zuwachs an erneuerbaren Energien seit 1998 zu einem guten zehnten Platz führt, reicht es bei der gesamten Stromerzeugung nur zu einem 23. Platz.

Diese Ergebnisse weisen darauf hin, dass die Ökosteuer im Verkehrssektor und das Erneuerbare-Energien-Gesetz (EEG) aus klimapolitischer Sicht ihre Wir-

Tabelle: Die Staaten mit den höchsten $\mathrm{CO}_{2}$-Emissionen

\begin{tabular}{lcc}
\hline & $\begin{array}{l}\text { Klimaschutz- } \\
\text { Index } \\
\text { Rangfolge }\end{array}$ & $\begin{array}{l}\text { Anteil an den } \\
\text { weltweiten } \\
\text { CO }_{2} \text {-Emissionen } \\
\text { (energiebedingt) }\end{array}$ \\
\hline Großbritannien & 3. & $2,2 \%$ \\
\hline Deutschland & 5. & $3,4 \%$ \\
\hline Indien & 10. & $4,2 \%$ \\
\hline China & 29. & $14,9 \%$ \\
\hline Japan & 34. & $4,8 \%$ \\
\hline Italien & 38. & $1,8 \%$ \\
\hline Kanada & 46. & $2,2 \%$ \\
\hline Russland & 48. & $6,1 \%$ \\
\hline Südkorea & 49. & $1,8 \%$ \\
\hline USA & 52. & $22,9 \%$ \\
\hline Summe & & $64,3 \%$ \\
\hline Quen & &
\end{tabular}

Quelle: eigene Darstellung kung nicht verfehlt haben. Im Gebäudesektor wird sich zeigen, wie wirksam die kürzlich beschlossenen Fördermaßnahmen für Gebäudedämmung und -sanierung sein werden. Wichtig für das zukünftige Abschneiden Deutschlands sind jedoch vor allem die anstehenden Entscheidungen bei den Kraftwerksneubauten. Der Neubau von Braunkohlekraftwerken könnte Deutschlands gesamte Klimapolitik konterkarieren. Auch ein Ausbau der Atomkraft kann kein Weg aus der Klimamisere sein. Deutschland kann seine Kohlendioxid-Emissionen im Stromsektor nur dann nachhaltig senken, wenn sowohl auf Produzenten-, als auch auf Verbraucherseite Maßnahmen getroffen werden. Zudem wird Investitionssicherheit für Energieeffizienz-Maßnahmen benötigt und Wind- Solar- und Biomasse-Anlagen müssen weiter massiv ausgebaut werden.

\section{Maßnahmen reichen nicht aus}

Der Klimaschutz-Index zeigt nur die relative Position der einzelnen Länder zueinander. Das bedeutet, dass auch diejenigen, die in der Rangliste gut abschneiden, sich nicht zurücklehnen dürfen. Sie gleichen eher den „Einäugigen unter den Blinden“. Um einen gefährlichen Klimawandel noch zu vermeiden, müssen bis Mitte des Jahrhunderts die Emissionen in Industrieländern um 80 Prozent und weltweit um 50 Prozent sinken. Die Politik muss dafür den Rahmen setzen. Der Klimaschutz-Index soll Transparenz und damit zusätzlichen Druck schaffen.

\section{Anmerkung}

(1) Die Publikation „Der Klimaschutzindex Vergleich der 53 Staaten mit dem größten $\mathrm{CO}_{2}$-Ausstoß" ist abrufbar unter www.germanwatch.org/ksi.htm

I AUTOREN + KONTAKT Jan Burck und Christoph Bals sind Mitarbeiter bei Germanwatch Deutschland.

Germanwatch, Dr. Werner-Schuster-Haus, Kaiserstr. 20, 53113 Bonn. Tel. 0228/60492-0, E-Mail: burck@germanwatch.org, bals@germanwatch.org 
(c) 20I0 Authors; licensee IÖW and oekom verlag. This is an article distributed under the terms of the Creative Commons Attribution Non-Commercial No Derivates License (http://creativecommons.org/licenses/by-nc-nd/3.o/), which permits unrestricted use, distribution, and reproduction in any medium, provided the original work is properly cited. 